\title{
FOTOINICIAÇÃO DE POLIMERIZAÇÃo VINÍLICA
}

\author{
Marcelo H. Gehlen*, Silmara F. Buchviser", Maira R. Rodrigues e Miguel G. Neumann* \\ Instituto de Química de São Carlos - Universidade de São Paulo - CP 780 - 13560-970 - São Carlos - SP
}

Recebido em 10/10/97; aceito em 24/3/98

\begin{abstract}
PHOTOINITIATION OF VINYL POLYMERIZATION. The initiation step of the light-induced polymerization kinetics of vinyl monomers using dye-sensitized photoinitiators to generate active radicals is discussed. The photoredox processes of basic dyes with amines and sulfinates are described as photochemical systems capable of starting free-radical polymerization of vinyl monomers in homogeneous and microheterogeneous media. Photophysical techniques like laser flash photolysis and time-correlated single photon counting are used to investigate the excited-state kinetics of the dyes.
\end{abstract}

Keywords: dyes; photoinitiators; radical polymerization.

\section{INTRODUÇÃO}

Nas últimas décadas o estudo de polimerização radicalar tem sido tema de inúmeras pesquisas em química, em parte devido à sua importância na fabricação de novos materiais. A maioria dos métodos industriais para obter polímeros são baseados em processos que se iniciam pela produção térmica de radicais livres. Grandes esforços tem sido dedicados à elucidação destas reações e, em geral, a cinética e os mecanismos são bem conhecidos ${ }^{1}$.

Por outro lado, alguns dos avanços tecnológicos mais importantes, como a fabricação de componentes micro-eletrônicos teriam sido impossíveis sem as técnicas de micro-litografia baseadas em fotopolimerização (photoresist techniques) ${ }^{2}$. Outras aplicações usuais da tecnologia de fotopolimerização incluem o uso de resinas fotopolimerizáveis em restaurações odontológicas e na produção de lentes oftalmológicas ${ }^{3,4}$. Aplicações modernas da tecnologia de fotopolimerização incluem também a produção de adesivos foto-sensíveis (fitas que se tornam aderentes quando irradiadas $)^{4}$, hologramas e a obtenção de objetos reais tridimensionais a partir de modelos ou imagens criados em computadores ${ }^{5,6}$. Todas estas aplicações requerem que a polimerização ocorra em regiões relativamente estreitas e bem localizadas. Essa condição não pode ser obtida usando os métodos habituais de polimerização térmica, já que nesses casos os processos ocorrem indiscriminadamente em toda a solução. A fotopolimerização requer a incidência de luz para a iniciação do processo, sendo que tal fato permite que a velocidade e localização espacial da reação de polimerização possa ser manipulada através do controle da intensidade, da energia e da distribuição espacial da luz. Este é um dos motivos pelos quais o interesse no estudo de polimerizações fotoiniciadas tem tido um crescimento significativo nesta última década.

Algumas das características desejáveis da radiação usada nestes processos é que ela seja absorvida por um dos componentes do sistema reacional, que possa ser obtida em altas intensidades, e colimada em regiões específicas da solução. Isto levou a um interesse crescente no desenvolvimento de novos sistemas de fotoiniciação sensíveis à luz visível, o que permitiria o uso de láseres para desencadear a polimerização ${ }^{5}$.

*Laboratório de Fotoquímica - http://www.iqsc.sc.usp.br

\# Endereço atual: Universidade Estadual de Ponta Grossa, PR
Na aplicação de láseres e outras fontes de alta intensidade, tais como os arcos de $\mathrm{Xe}$ e $\mathrm{W}$ que emitem intensamente na região do visível $(420-650 \mathrm{~nm})$, os sistemas fotoquímicos terão que incluir necessariamente iniciadores que absorvam nessa faixa espectral. Corantes orgânicos são uma escolha adequada para estas finalidades, devido a seus altos coeficientes de extinção (>20.000 $\mathrm{M}^{-1} \mathrm{~cm}^{-1}$ ) e propriedades químicas, tais como serem bons redutores e oxidantes em seus estados excitados. Por outro lado, podem ser escolhidos corantes que absorvam luz seletivamente a diferentes comprimentos de onda, como se pode ver na figura 1 . Sistemas contendo corantes tem sido utilizados para iniciar fotoquimicamente vários tipos de polimerizações, especialmente aquelas iniciadas por radicais livres $^{7-13}$.

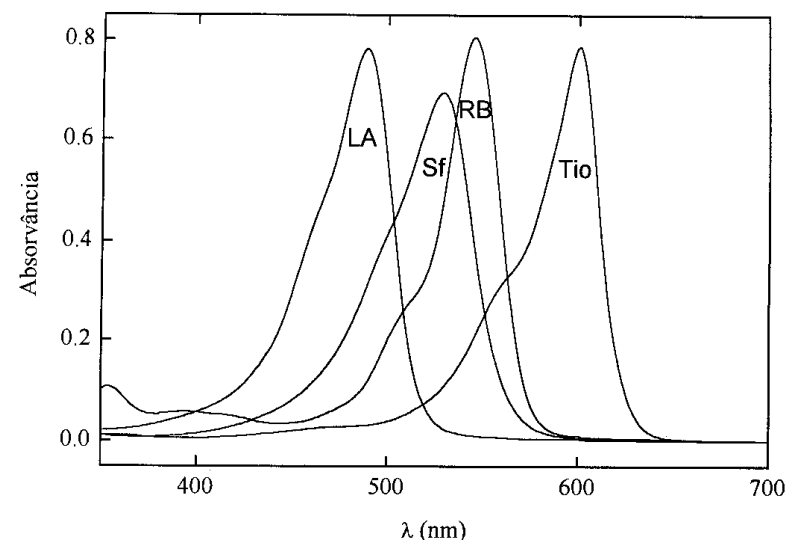

Figura 1. Espectros de absorção de corantes usados em fotoiniciação de polimerização. LA: Laranja de acridina $1,13 \times 10^{-5} \mathrm{M}$, Sf: Safranina $1,12 \times 10^{-5} \mathrm{M}$, RB: Rodamina B $0,56 \times 10^{-5} \mathrm{M}$, e Tio: Tionina $0,8 \times 10^{-5} \mathrm{M}$.

Apesar da relativa maturidade de alguns aspectos do campo da fotopolimerização, estudos fotofísicos e fotoquímicos detalhados das fotorreações de sistemas iniciadores ainda são relativamente escassos, principalmente em meios organizados. Neste trabalho, pretendemos exemplificar os tipos de processos envolvidos nas polimerizações fotoiniciadas, e mostrar como a partir de informações correspondentes às reações individuais é possível predizer o comportamento das reações de polimerização global.

Embora existam várias possibilidades de fotoiniciação de reações de polimerização, a discussão será centrada na iniciação radicalar de polimerização vinílica em meio homogêneo (solvente) 
e microheterogêneo (micro-emulsão), com corantes como iniciadores.

\section{CINÉTICA DE FOTOINICIAÇ̃̃o DE POLIMERIZAÇÃO RADICALAR}

\section{i) Sistemas Homogêneos}

As reações de fotopolimerização são de dois tipos básicos: reações de foto-entrelaçamento (photo-crosslinking, ou curing), nas quais a irradiação provoca a formação de ligações cruzadas entre polímeros pré-formados, dando materiais de maior massa molecular, e polimerizações em cadeia fotoiniciadas. O processo em cadeia apresenta três estágios: (a) iniciação; (b) propagação da cadeia; e (c) terminação da cadeia ${ }^{1-4}$. Ao contrário das reações de polimerização iniciadas termicamente, as reações de fotopolimerização podem ser conduzidas a temperatura ambiente.

O mecanismo geral de polimerização vinílica por radicais livres está mostrado na figura 2 , onde In corresponde ao iniciador, $\mathbf{R}^{\bullet}$ a radicais livres, $\mathbf{M}$ ao monômero, e $\mathbf{M}_{\mathbf{n}}$ ao polímero. As reações (a-e) são as de iniciação, transferência de radicais, propagação, e terminação por recombinação e por disproporcionamento, respectivamente. Na polimerização térmica, os radicais livres que iniciam o processo são obtidos geralmente por decomposição de azo-compostos ou peróxidos, como indicado nas eqs 1 e 2 .

$$
\begin{aligned}
&\left(\mathrm{CH}_{3}\right)_{3} \mathrm{C}-\mathrm{N}=\mathrm{N}-\mathrm{C}\left(\mathrm{CH}_{3}\right)_{3} \rightarrow \mathrm{N}_{2}+2\left(\mathrm{CH}_{3}\right)_{3} \mathrm{C}^{\bullet} \\
&\left(\mathrm{CH}_{3}\right)_{3} \mathrm{C}-\mathrm{O}-\mathrm{O}-\mathrm{C}\left(\mathrm{CH}_{3}\right)_{3} \rightarrow \rightarrow 2\left(\mathrm{CH}_{3}\right)_{3} \mathrm{CO}^{\bullet} \rightarrow \\
& 2 \mathrm{CH}_{3}+\underset{\mathrm{CH}_{3} \mathrm{COCH}_{3}}{\longrightarrow}
\end{aligned}
$$

Usando os critérios de estado estacionário e supondo que as constantes de velocidade para as reações de propagação não dependem do comprimento da cadeia, pode ser obtida uma expressão para a velocidade de polimerização térmica ${ }^{1}$

$$
\begin{aligned}
& \mathrm{v}_{\mathrm{p}}=k_{p}\left(k_{i} / k_{t}\right) \gamma_{2[M][\ln \gamma} \gamma_{2} \\
& \mathrm{In} \stackrel{K_{i} \text { ou } h \mathrm{v}}{\longrightarrow} \mathrm{R}^{¥} \\
& \mathrm{R} ¥+\mathrm{M} \stackrel{k_{r}}{\longrightarrow} \mathrm{RM} ¥ \\
& \mathrm{RM}^{¥}+\mathrm{nM} \stackrel{k_{p}}{\longrightarrow} \mathrm{RM} ¥ \\
& \mathrm{RM}_{\mathrm{n}}^{¥}+\mathrm{RM}_{\mathrm{m}}^{¥} \stackrel{k_{t c}}{\longrightarrow} \mathrm{RM}_{(\mathrm{n}+\mathrm{m})} \\
& \mathrm{RM}_{\mathrm{n}}^{\mathrm{Z}}+\mathrm{RM} \mathrm{R}_{\mathrm{m}} \stackrel{k_{t d}}{\longrightarrow} \mathrm{RM}_{\mathrm{n}} \mathrm{H}+\mathrm{RM}_{\mathrm{n}}(-\mathrm{H})
\end{aligned}
$$

Figura 2. Esquema reacional para polimerização vinílica por radicais livres.

Em sistemas nos quais a iniciação é fotoquímica, podem ocorrer duas situações distintas ${ }^{7,12}$. No primeiro caso, denominado de unimolecular, a produção de radicais livres é direta e resultante da cissão homolítica / heterolítica do iniciador fotoquímico, tal como descrito nas eqs 1 e 2 . A reação de decomposição fotoquímica de azoisobutironitrila é um exemplo destes sistemas e, em geral, os mesmos iniciadores de polimerização térmica (e.g., peróxidos, azocompostos, compostos halogenados e carbonílicos) podem ser decompostos fotoquimicamente. Neste caso, a velocidade de polimerização tem uma dependência direta com a intensidade da luz absorvida $I_{a}$ e com o rendimento quântico $\phi_{d}$ de fotodecomposição do iniciador

$\mathrm{v}_{\mathrm{p}}=k_{p}\left(l_{a} \phi_{d} / k_{t}\right)^{y^{2}}[\mathrm{M}]$

O segundo caso, chamado de iniciação bimolecular, envolve sistemas nos quais o iniciador (corantes ou cetonas aromáticas) é excitado eletronicamente, reagindo posteriormente com um coiniciador (aminas alifáticas, arilsulfinatos), levando à formação de radicais livres que desencadeiam a polimerização. Para o caso de corantes

$\mathrm{D}+\mathrm{h} v \rightarrow \mathrm{D}^{*}$

$\mathrm{D}^{*}+\mathrm{CoIn} \rightarrow \rightarrow \mathrm{R}^{\bullet}+$ Produtos

onde $\mathrm{D}$ e $\mathrm{D}^{*}$ correspondem ao corante nos estados fundamental e excitado, e CoIn é o co-iniciador.

Neste caso a velocidade de polimerização depende da eficiência de formação de radicais livres a partir dos estados excitados do iniciador. Esta eficiência, denominada de $\eta$, é função de todos os processos elementares envolvidos na eq 6 . Desta forma, a velocidade de polimerização fotoiniciada é dada por ${ }^{8,9}$

$\mathrm{v}_{\mathrm{p}}=k_{p}\left(I_{\mathrm{a}} \eta / k_{\mathrm{q}}\right)^{1 / 2}[M]$

A intensidade de luz absorvida está relacionada com a absorvância do sistema e a intensidade de luz incidente $I_{o}$ por:

$I_{a}=I_{o}\left(1-10^{-\mathrm{Abs}}\right)$

onde $\mathrm{Abs}=\varepsilon \lambda \ell[\mathrm{In}]$, i.e., o produto do coeficiente de extinção molar do iniciador no comprimento de onda de excitação vezes o percurso óptico $\ell$ e concentração molar do iniciador.

\section{ii) Sistemas Coloidais}

A polimerização radicalar com iniciação térmica ou fotoquímica em sistemas micro-heterogêneos tem sido um campo paralelo de pesquisa e desenvolvimento na área de polímeros. Podemos incluir neste campo, a polimerização em emulsão, microemulsão e outros sistemas micelares ${ }^{14-16}$.

Um sistema típico de polimerização em emulsão consiste em uma solução aquosa contendo um monômero pouco solúvel, um emulsificante (surfactante) e o iniciador. O sistema reacional é formado de gotículas de monômero (de 1-10 $\mu$ de diâmetro) estabilizadas pelo surfactante, bem como de micelas (de 2-20 nm) contendo uma fração reduzida de monômero solubilizado. No caso de sistemas de emulsões reversas de água em óleo, o meio de solubilização é um solvente apolar, os monômeros são hidrofílicos, e as micelas formadas são estruturas reversas.

Sistemas denominados de microemulsões normais ou reversas consistem em uma fase líquida contendo somente micelas saturadas de monômero, sendo formadas quando as concentrações molares de surfactante e monômero são da mesma ordem ${ }^{16}$.

Iniciadores térmicos tem sido frequentemente utilizados em polimerização em emulsão. Uma certa restrição ao uso de iniciadores fotoquímicos ocorre devido ao forte espalhamento de luz que é observado em soluções coloidais, contendo partículas da ordem de micra. Contudo, em microemulsões normais ou reversas, onde a dimensão das micelas e partículas formadas são menores que $100 \mathrm{~nm}$, temos a formação de soluções completamente límpidas permitindo amplamente a aplicação de métodos fotoquímicos na iniciação de polimerização ${ }^{17-19}$.

A polimerização em emulsão ou microemulsão tem várias 
vantagens destacando-se: (1) a excelente dissipação térmica, devido ao sistema permanecer líquido durante o transcurso da reação exotérmica de polimerização; (2) a alta velocidade de polimerização; (3) a obtenção de polímeros de elevado peso molecular. $\mathrm{O}$ produto final encontra-se na forma de um látex que pode ser diretamente utilizado em aplicações industriais tais como a produção de tintas, adesivos e elastômeros ${ }^{16}$. No caso de polimerização em microemulsão, existe a possibilidade de obtenção de partículas nanométricas praticamente monodispersas, as quais encontram aplicação na formulação de novos materiais poliméricos.

A polimerização em emulsão ocorre em três etapas distin$\operatorname{tas}^{14,15,20}$. O estágio inicial envolve a formação de partículas de látex no interior das micelas devido à migração de radicais livres gerados na fase aquosa para a fase micelar. $O$ equilíbrio de solubilização é mantido pela difusão de monômeros das gotículas (que funcionam como um reservatório) para as micelas. Neste estágio inicial tem-se uma conversão 0-10 \%. Ao final da nucleação, inicia-se o segundo estágio no qual o número de partículas de látex estabilizadas com o surfactante permanece constante. A taxa de polimerização resulta em um crescimento das partículas de látex, e a concentração local de monômero permanece constante enquanto existirem em solução as gotículas ricas em monômero. Esta etapa compreende uma conversão 10-40 \% do monômero em polímero. O terceiro estágio é caracterizado pelo desaparecimento das gotículas de monômero e uma etapa de polimerização final com decréscimo na concentração de monômero nas partículas de látex. A velocidade de polimerização $v_{p}$ e o grau de polimerização $D P$ são dados por:

$v_{p}=k_{p} \bullet n \circledR[\mathrm{M}][\mathrm{L}]$

$D P=2 k \bullet n \circledast[\mathrm{M}][\mathrm{L}] / \mathrm{v}_{i}$

onde [L] é a concentração de partículas de látex, $\bullet$ ® é o número médio de radicais por partícula de látex, $[\mathrm{M}]$ é a concentração de monômero, e $k_{p}$ é a constante de polimerização. A velocidade de geração de radicais $v_{i}$, para um processo de iniciação fotoquímica é dado por $v_{i}=\eta I_{a}$. A cinética de polimerização descrita pela eq 9 fica inteiramente determinada pelo número médio de radicais $\bullet ~ n \circledR$ por partícula de látex nas diversas fases do processo. A formulação cinética para o cálculo de - $n \circledR$ foi descrita primeiramente por Smith e Ewart ${ }^{20}$. Devido à característica estatística do número de radicais por partícula, a polimerização em sistemas de confinamento molecular requer um tratamento estocástico para o cálculo de $\bullet n 囚$, com aplicação de um formalismo cinético similar ao utilizado frequentemente para descrever reações de estados excitados em sistemas micelares $^{21}$.

No modelo de Smith e Ewart, o número de partículas com $n$ radicais, $\mathrm{L}_{\mathrm{n}}$, é vinculado ao seguinte esquema cinético,

$\mathrm{R}+\mathrm{L}_{\mathrm{n}} \mathrm{L}_{\mathrm{n}+1} \underset{\mathrm{k}_{-}}{\stackrel{\mathrm{k}_{+}}{\rightleftharpoons}}(n=0,1,2, \ldots)$

$L_{n} \quad L_{n-2} \stackrel{\mathrm{n}(\mathrm{n}-1) \mathrm{k}_{\mathrm{t}}}{\longrightarrow}(n=2,3, \ldots)$

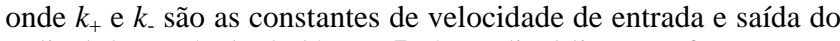
radical da partícula de látex e $\mathbf{R}$ é o radical livre em fase aquosa. $k_{t}$ é a constante de velocidade de terminação ou recombinação de dois radicais na mesma partícula de látex. A equação de velocidade correspondente ao esquema cinético é dada por:

$$
\begin{array}{rl}
\frac{\mathrm{d} L_{n}}{\mathrm{dt}}=k_{+} A\left(L_{n-1}+L_{n}\right)+k & k\left((n+1) L_{n+1}-n L_{n}\right)+ \\
\left.\left.t(k n+2)(n-1) L_{n+2}-n(n-1) L_{n}\right)\right)
\end{array}
$$

sendo que o valor médio é calculado por: $\{\mathrm{n}\rangle=\sum \mathrm{nL}_{\mathrm{n}}$

A equação de velocidade para o processo de recombinação de radicais no interior da partícula de látex (eq 13) é basicamente o mesmo de um processo de aniquilação triplete-triplete em sistemas micelares com migração ${ }^{22}$. A diferença está na condição inicial e na de contorno do problema. No processo de recombinação de radicais assume-se que no início as partículas não contém radicais, isto é, $L_{n}(0)=\delta_{0, n}$. A concentração de radicais na fase aquosa é mantida constante pela geração térmica ou fotoquímica. Uma solução analítica no caso geral não é conhecida. Contudo no regime estacionário (referente ao segundo estágio) onde $L_{n}$ é constante, o número médio de radicais por partícula é dado por ${ }^{23}$

$i n '=\frac{s I_{r}(s)}{4 I_{r-1}(s)}$

onde $r=k / k_{t}, s=2\left(k_{+}[\mathrm{R}]_{a q} / k_{t}\right)^{12}, e I_{r}(s)$, é a função modificada de Bessel de $1^{\mathrm{a}}$ classe de ordem $r$. Quando a velocidade de recombinação é muito mais rápida que a velocidade de migração, $\bullet n ® 0,5$, o que significa que as partículas de látex contém uma ou nenhuma cadeia de propagação, sendo esta uma situação clássica do modelo de Smith e Ewart ${ }^{20}$.

Estudos recentes tem demonstrado que o processo de nucleação homogênea proveniente de radicais gerados na fase aquosa não explica completamente a dispersão de tamanho de partículas obtidos por medidas de espalhamento de luz. Os resultados indicaram que a nucleação e o crescimento das partículas de látex ocorrem em paralelo por um mecanismo de coagulação ${ }^{24}$.

Embora a descrição original do modelo de Smith e Ewart descreva um processo bimolecular de recombinação de radicais para a terminação, em certos sistemas pode ocorrer a presença de processos de transferência de cadeia para aditivos, tais como emulsificantes e surfactantes, gerando radicais pouco ativos. Hunkeler e colaboradores ${ }^{25}$ utilizaram um modelo modificado para a polimerização em emulsão reversa de acrilamida incluindo terminação unimolecular ou monoradicalar e um processo de migração de oligômeros. Os resultados experimentais puderam ser descritos muito satisfatoriamente pelo modelo teórico proposto.

A polimerização em microemulsão, diferente ao que ocorre no processo em emulsão, tem uma cinética caracterizada pela nucleação contínua e formação de partículas de látex de dimensões reduzidas similar à dimensão das micelas originais que contém o monômero ${ }^{16}$. Por ser uma técnica nova de polimerização, estudos teóricos descrevendo a cinética são escassos. Contudo, resultados experimentais tem demonstrado que a velocidade de polimerização segue uma equação empírica do tipo $\mathrm{R}_{\mathrm{p}}=k h^{\alpha}[\mathrm{M}]^{\beta}$ sendo $\alpha$ e $\beta$ expoentes característicos para um determinado sistema. Por exemplo, na fotopolimerização de acrilamida em microemulsões de aerosol AOT em tolueno usando como iniciador AIBN, o processo de terminação é monoradicalar, proveniente da transferência degradativa de cadeia ao solvente tolueno, formando radicais benzila que são razoavelmente estáveis ${ }^{26,27}$. Neste caso, a velocidade de polimerização segue a equação

$\mathrm{v}_{\mathrm{p}}=\mathrm{k}_{\mathrm{p}}[\mathrm{M}] \frac{2 f_{\mathrm{r}_{\mathrm{a}}} \mathrm{I}_{\mathrm{In}}[\mathrm{I}]}{\mathrm{k}_{\mathrm{t}}[\mathrm{T}]}$

onde $k_{t}$ é a constante de terminação monoradicalar e [T] é a concentração do agente de terminação. A polimerização em microemulsão apresenta vantagens e desvantagens com relação ao processo em emulsão. Em microemulsão obtêm-se partículas de 
látex com tamanhos da ordem da micela original, ou seja, partículas nanométricas praticamente monodispersas e contendo uma macromolécula por partícula. No transcurso da reação, o sistema microheterogêneo é estável e transparente. A desvantagem provém do uso de altas concentrações de surfactante e estabilizantes, o que dificulta processos de separação do produto final, bem como um aumento do custo na produção de um polímero. Contudo, a obtenção de polímeros hidrossolúveis via polimerização em micelas reversas resulta em um produto final já parcialmente hidratado, que permite sua solubilização praticamente instantânea em água, diferindo do polímero em fase sólida que usualmente gelifica e exige agitação mecânica intensa para a formação de uma solução.

A copolimerização em emulsão e microemulsão com iniciação fotoquímica tem sido bastante investigada, ${ }^{15,18,19}$ porém modelos cinéticos para processos envolvendo mais de um tipo de monômero são questões pouco exploradas neste campo de pesquisa.

\section{SISTEMAS E MECANISMOS FOTOQUÍMICOS ENVOLVENDO CORANTES}

Em geral, a presença de um corante que absorve a luz e de um co-iniciador (ver Figuras 3 e 4), leva a algumas modificações cinéticas das reações descritas na figura 2 .

Nesta nova situação deverão ser consideradas as conversões entre os estados excitados do corante e seu decaimento ao estado fundamental, as reações de supressão dos estados singlete e triplete tanto pelo co-iniciador como pelo monômero, e os equilíbrios ácido-base do co-iniciador e do corante nos estados eletrônicos excitados. Devido à sua configuração eletrônica, com um elétron no orbital LUMO e uma vacância no orbital HOMO, o corante no estado excitado é bastante reativo e melhor redutor ou oxidante que o mesmo no estado fundamental. Portanto, depois de excitados, os corantes geralmente tem condições termodinâmicas favoráveis para a abstração (ou doação) de um elétron de um doador (ou aceptor) apropriado presente na solução. Estes doadores e aceptores de elétrons (ver Figura 4) são chamados de co-iniciadores e devem estar presentes na mistura reacional para a formação de radicais livres.

Um esquema simplificado das reações que são consideradas em um sistema de polimerização fotoiniciada em presença de um corante D e um doador de elétrons A é apresentado na figura 5. Quando em presença de doadores de elétrons, como aminas alifáticas terciárias, os corantes catiônicos no estado triplete abstraem um elétron do co-iniciador para formar as espécies semirreduzidas do corante e um radical cátion da amina. Em geral, as espécies semirreduzidas não são muito eficientes para a iniciação da polimerização. Pelo contrário, podem agir como agentes terminadores de cadeia. Por outro lado, a iniciação pela espécie derivada do corante também não é desejável, já que isso poderia resultar em consumo gradual do corante diminuindo a taxa de absorção de radiação.

Os co-iniciadores ideais devem apresentar uma série de propriedades, tais como: potencial de oxidação (ou de redução) relativamente alto, para uma doação (ou abstração) eficiente de elétron no processo redox com o corante excitado; alta reatividade na adição do radical à dupla ligação; não absorver luz no mesmo comprimento de onda de irradiação do corante; não reagir nem interagir com o corante ou com os monômeros no estado fundamental; suprimir pouco o estado singlete excitado do corante, visto que uma desativação fotofísica não conduz a radicais livres.

\section{i) Corante / Amina}

Para ilustrar os processos envolvidos e as reações que devem ser consideradas em um caso específico, analisaremos o processo da fotoiniciação de polimerização em metanol de
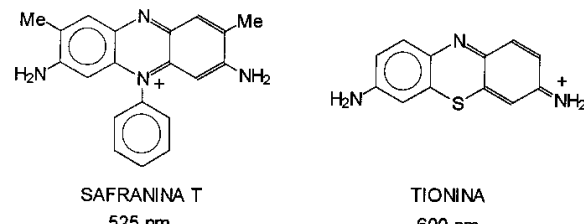

$525 \mathrm{~nm}$
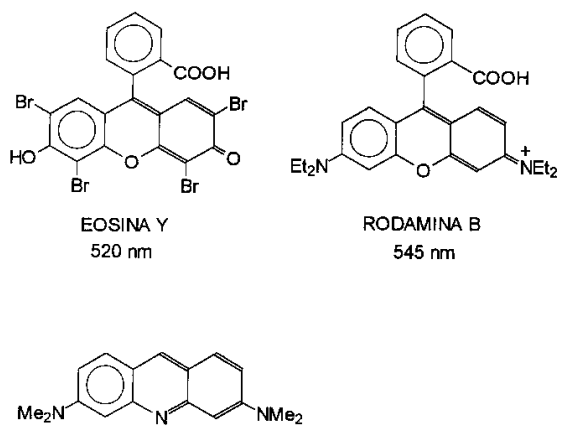

LARANUA DE ACRIDINA

$490 \mathrm{~nm}$

Figura 3. Corantes usados como fotoiniciadores e seus máximos de absorção na região visível.

\section{CO-INICIADORES}

$\underline{\text { Doadores de el•trons }}$

Etanolaminas

Aminas alifłticas terciłrias

Aminas arom $\neq$ ticas terciłrias

Arsinas aromłticas

Arilsulfinatos

Compostos heteroc'clicos

Organo-boratos

$$
\begin{aligned}
& \left(\mathrm{HOCH}_{2} \mathrm{CH}_{2}\right)_{3} \mathrm{~N} \\
& \mathrm{R}_{3} \mathrm{~N} \\
& \mathrm{Ph}_{3} \mathrm{~N} \\
& \mathrm{Ph}_{3} \mathrm{As} \\
& \mathrm{RPhSO}_{2}{ }^{-} \\
& \text {oxaz-is, tiaz-is, e } \\
& (\mathrm{Ph})_{3} \mathrm{BR}
\end{aligned}
$$

Aceptores de el•trons

Sais de arildiaz ${ }^{\mathrm{TM}}$ nio

Sais de diariliod ${ }^{\mathrm{TM}}$ nio

Sais de fosf ${ }^{\mathrm{TM}}$ nio

Hidrocarbonetos polihalogenados

$\mathrm{ArN}_{2}{ }^{+} \mathrm{X}^{-}$
$\mathrm{Ar}_{2} \mathrm{I}^{+} \mathrm{MX}^{-}$
$\mathrm{Ar}_{3} \mathrm{P}^{+}-\mathrm{CH}_{2} \mathrm{RX}^{-}$
$\mathrm{RX}_{\mathrm{n}}$

Figura 4. Co-iniciadores usados junto com os corantes para polimerização fotoiniciada.

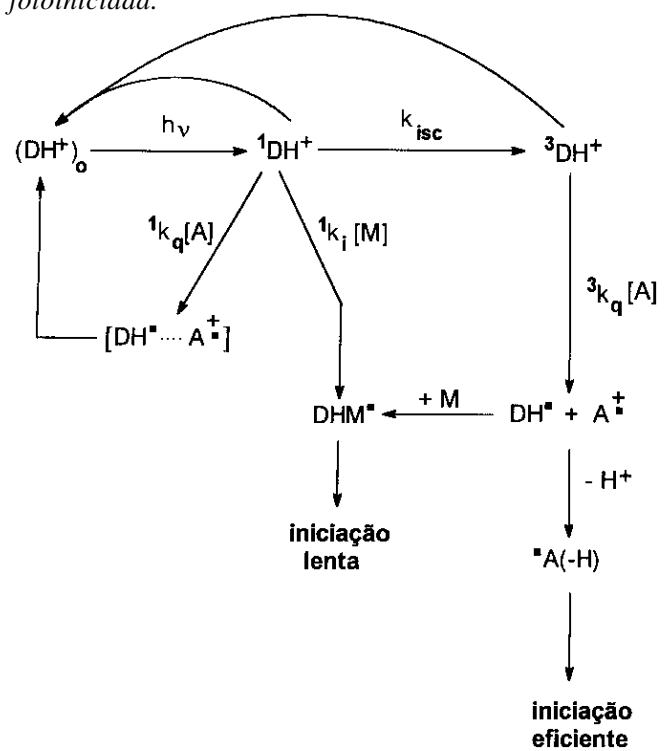

Figura 5. Mecanismo de polimerização vinílica iniciada por corantes. 
2-hidroxietilmetacrilato $\mathrm{H}_{2} \mathrm{C}=\mathrm{C}\left(\mathrm{CH}_{3}\right) \mathrm{CO}_{2} \mathrm{CH}_{2} \mathrm{CH}_{2} \mathrm{OH}(\mathrm{M})$ pelo corante safranina $\mathrm{T}\left(\mathrm{SfH}^{+}\right)$em presença de trietanolamina $\mathrm{N}\left(\mathrm{CH}_{2} \mathrm{CH}_{2} \mathrm{OH}\right)_{3}(: \mathrm{Am})^{30}$.

Em primeiro lugar temos que analisar as possíveis reações entre as espécies presentes no sistema no estado fundamental. Estas incluem a auto-agregação do corante (eq 17), e as interações entre corante e amina, corante e monômero, e amina e monômero.

$\mathrm{SfH}++\mathrm{SfH}+\approx E\left(\mathrm{SfH}^{+}\right) \stackrel{+\mathrm{nSfH}^{*}}{\longrightarrow}\left(\mathrm{SfH}^{+}\right)$

Os corantes quando em solução moderadamente concentrada podem sofrer uma auto-agregação evidenciada inequivocamente pela variação no espectro de absorção do sistema ${ }^{31-33}$. No presente caso, este processo não ocorre pois o espectro uv-vis da solução não apresenta novas bandas, ou modificações vinculadas a dímeros ou agregados maiores. De forma similar, a invariança do espectro de absorção do corante na presença de monômero ou de aminas indica que não há interação no estado fundamental.

Uma vez irradiada a solução, o corante é inicialmente levado ao primeiro estado excitado singlete.

$$
\left(\mathrm{SfH}^{+}\right) \stackrel{\mathrm{H}}{\longrightarrow}{ }^{1} \mathrm{SfH}^{+}
$$

Os principais processos que afetam o estado excitado são: decaimento ao estado fundamental por emissão de um fóton (fluorescência) ou dissipação térmica de energia (conversão interna) e a conversão para o estado triplete. Na ausência de supressores, o tempo de vida do estado singlete da safranina é 2,3 ns (em metanol), e a eficiência de sua conversão ao triplete é de aproximadamente $20 \%{ }^{34-38}$. Assim, para otimizar o processo devemos evitar que a reação de desativação do estado singlete do corante pela amina, ou pelo monômero, diminuam a quantidade de triplete formado. A reação entre o estado singlete e a amina forma um complexo de transferência de carga (eq 19), que por conservação de momento de spin na coordenada de reação, conduz preferencialmente para o estado fundamental do corante (eq 20), com uma produção reduzida de radicais livres (eq 21)

$$
\begin{aligned}
& { }^{1} \mathrm{SfH}^{+}+: \mathrm{Am} \longrightarrow{ }^{1}\left(\mathrm{SfH}^{+} \ldots \mathrm{Am}\right) \rightarrow\left[\mathrm{SfH}^{\delta+} \ldots{ }^{\delta-} \mathrm{Am}\right] \\
& {\left[\mathrm{SfH}^{\delta+} \ldots{ }^{\delta-} \mathrm{Am}\right] \longrightarrow\left(\mathrm{SfH}^{+}\right)_{0}+: \mathrm{Am}} \\
& {\left[\mathrm{SfH}^{\delta+} \ldots{ }^{\delta-} \mathrm{Am}\right] \longrightarrow \mathrm{SfH}^{\cdot}+{ }^{\bullet} \mathrm{Am}}
\end{aligned}
$$

Estudos da supressão da fluorescência da safranina por trietanolamina permitiram determinar que a constante de supressão é de $4,1 \times 10^{9} \mathrm{M}^{-1} \mathrm{~s}^{-1}$, um valor próximo ao limite difusional em metanol ${ }^{35-37}$. Portanto, para concentrações de amina maiores que $1 \mathrm{M}$ praticamente não se forma uma população apreciável de estado triplete. Isto estabelece um limite superior para a concentração que deva ser usada do co-iniciador. Por outro lado, não foi observado experimentalmente um aumento da velocidade de polimerização com o aumento da concentração de co-iniciador. Ao contrário, observou-se um decréscimo desta velocidade a partir de certa concentração de amina, confirmando que a interação do estado singlete com a trietanolamina não leva a uma eficiente produção de radicais iniciadores de polimerização. No presente sistema, não foi observada supressão da fluorescência da safranina pelo monômero. Isto indica que a etapa de fotoiniciação do sistema estudado ocorre a partir do estado excitado triplete reagindo com a trietanolamina.

A fotorredução do corante envolve o estado triplete devido ao tempo de vida mais longo desta espécie (em torno de $15 \mu \mathrm{s}$, dependendo do solvente). No caso específico da safranina, existe um equilíbrio prototrópico no estado excitado triplete ${ }^{34-37}$.

${ }^{3} \mathrm{SfH}^{+} \longrightarrow \mathrm{Sf}+\mathrm{H}^{+}$ com $\mathrm{pK}_{\mathrm{a}}$ diferente do estado fundamental. Assim, o primeiro processo do estado triplete será sua desprotonação com transferência do próton para a amina

${ }^{3} \mathrm{SfH}^{+}+: \mathrm{Am} \longrightarrow{ }^{3} \mathrm{Sf}+: \mathrm{AmH}^{+}$

Através da técnica de fotólise por pulso de laser foi comprovado que a velocidade de supressão do estado triplete da safranina protonada tem uma constante bimolecular de $1,3 \times 10^{7}$ $\mathrm{M}^{-1} \mathrm{~s}^{-1}$, o que é alto demais para um processo de transferência de elétron quando comparado com a velocidade de supressão do estado singlete ${ }^{35,36}$. No mecanismo cinético, o triplete desprotonado é posteriormente suprimido pela amina via transferência de elétron similar ao do estado singlete (eq 24), mas com a diferença que neste caso a produção de radicais livres (eq 25) é muito mais eficiente que o decaimento ao estado fundamental.

$$
\begin{aligned}
& { }^{3} \mathrm{Sf}+: \mathrm{Am} \longrightarrow{ }^{3}[\mathrm{Sf} . . \mathrm{Am}] \longrightarrow\left[\mathrm{Sf}^{\delta-} \ldots{ }^{\delta+} \mathrm{Am}\right] \\
& {\left[\mathrm{Sf}^{\delta-} \ldots{ }^{\delta+} \mathrm{Am}\right] \longrightarrow \mathrm{SF}^{{ }^{-}}+{ }^{\cdot} \mathrm{Am}^{+}}
\end{aligned}
$$

O cátion radical da amina perde rapidamente um próton dando lugar ao radical que efetivamente inicia o processo de polimerização

$\cdot \mathrm{Am}^{+} \rightarrow \cdot \mathrm{Am}+\mathrm{H}^{+}$

Com estas avaliações é possível postular uma expressão de estado fotoestacionário para a velocidade de produção de radicais livres ${ }^{30}$

$\Phi_{\text {rad }}=\frac{\Phi_{\text {isc }}}{1+{ }^{1} K_{S V}[\mathrm{Am}]} \cdot \frac{{ }^{3} K_{S V}^{p}[\mathrm{Am}]}{1+{ }^{3} K_{S V}^{p}[\mathrm{Am}]} \cdot \frac{{ }^{1} K_{S \zeta}^{p}[\mathrm{Am}]}{1+{ }^{3} K_{S V}^{d}[\mathrm{Am}]}$

onde as $K_{S V}\left(=k_{q} \cdot \tau\right)$ representam as constantes de Stern-Volmer para supressão dos estados singlete e triplete protonado e desprotonado pela amina, e $\phi_{i s c}$ é o rendimento quântico de formação de triplete na ausência de supressores.

Na figura 6 está representada a velocidade inicial de polimerização em função da concentração de trietanolamina na solução. A curva corresponde a avaliação feita usando-se a eq 27 enquanto que os pontos são experimentais. Os valores das $K_{S V}$ foram determinados anteriormente para sistemas que não incluíam monômeros e os valores usados foram ${ }^{1} K_{S V}=7,4 \mathrm{M}^{-1}$, ${ }^{3} \mathrm{~S}$ $=390 \mathrm{M}^{-1}$, e $\mathcal{K}_{\mathrm{SV}}^{\mathrm{d}}=30 \mathrm{M}^{-1}$.

Outros corantes catiônicos, como a tionina, podem ser utiliza-

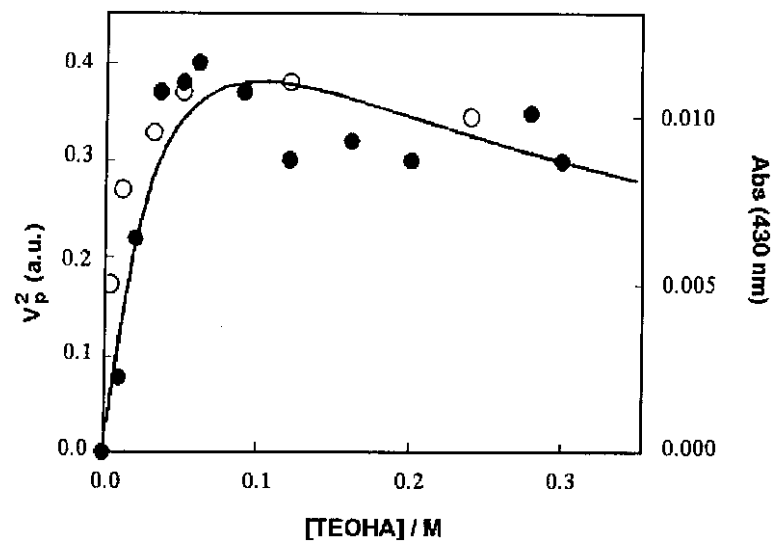

Figura 6. Gráfico do quadrado da velocidade de polimerização (•) $e$ da densidade óptica a tempos longos (O) em função da concentração de trietanolamina. A curva corresponde ao cálculo utilizando a Eq. 27. 
dos como fotoiniciadores na reação redox com aminas. Por exemplo, a reação entre a tionina no estado triplete com trietanolamina segue a cinética similar à da safranina com formação de $\alpha$ aminoradicais (eqs 24-26). O estado triplete é suprimido pela amina em uma reação rápida com constante de velocidade da ordem de $1,6 \times 10^{7} \mathrm{M}^{-1} \mathrm{~s}^{-1} 39$. O decaimento de triplete da tionina na ausência e presença de trietanolamina obtido pela técnica de fotólise por pulso de laser é apresentado na Figura 7, mostrando a supressão do corante excitado pelo co-iniciador.

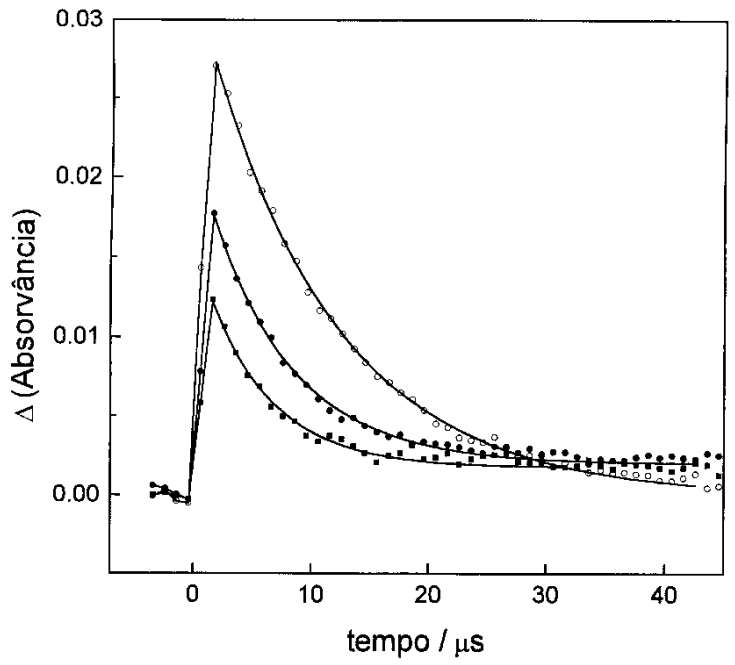

Figura 7. Decaimento do estado triplete de tionina em metanol obtido por fotólise de pulso de laser, na ausência e presença de trietanolamina (घ; 0, • 2.0, $\mathbf{\Delta} ; 2,5 \mathrm{mM}$ ). Transientes observados em $720 \mathrm{~nm}$.

\section{ii) Corante / sulfinato}

Arilsulfinatos são co-iniciadores efetivos para polimerização radicalar onde corantes catiônicos atuam como fotoiniciadores em um processo redox ${ }^{7-9,40-44}$. Estudos de supressão de fluorescência, medidas de rendimento quântico e estudos de fotólise estabeleceram que o processo de iniciação pode ser representado pelo seguinte esquema cinético

$\mathrm{D}^{+}+\mathrm{ArSO}_{2}^{-} \rightarrow \mathrm{D}^{+}+\mathrm{ArSO}_{2}^{\circ}$

$\mathrm{ArSO}_{2}^{\circ}+\mathrm{M} \longrightarrow \mathrm{ArSO}_{2} \mathrm{M}^{\circ}$

Embora a reação de formação de radicais via triplete seja mais eficiente, em concentrações elevadas de sulfinato o processo via estado singlete também contribui. Os radicais $\mathrm{ArSO}_{2}{ }^{*}$ são muito reativos, podendo ser utilizados na polimerização de derivados de estireno. Contudo neste caso os monômeros vinílicos derivados de estireno suprimem eficientemente o estado singlete de corantes catiônicos sem a geração de radicais ativos ${ }^{43}$. Esta reação competitiva reduz a eficiência global da etapa de iniciação e formação de radicais sulfinato, afetando marcadamente a velocidade de polimerização. O decaimento de fluorescência de um corante catiônico (acridina) na ausência e presença de um derivado de estireno é ilustrado na figura 8. As constantes bimoleculares de desativação de corantes catiônicos por derivados de estireno são da ordem do limite difusional, ocasionando uma eficiência muita baixa do sistema iniciador fotoquímico em polimerização em fase homogênea ${ }^{43}$.

Uma das alternativas para aumento da eficiência da etapa de iniciação fotoquímica é confinar o sistema iniciador no interior de uma micela reversa visto que ambas as espécies corante e coiniciador neste caso são íons ${ }^{43,44}$. Neste procedimento existem duas vantagens. A baixa solubilidade do monômero na microfase aquosa, reduz em uma a duas ordens de grandeza a taxa de supressão de fluorescência. Tal fato fica evidente no gráfico comparativo de intensidade relativa em função da concentração de monômero em meio homogêneo e em meio micelar reverso no caso do sistema acridina / estireno conforme visto na figura 9. Para este sistema, a razão entre a constante de supressão na fase micelar reversa e na fase homogênea (metanol) tem o valor de 0,06 . Por outro lado, o confinamento molecular de corante e co-iniciador na estrutura nanométrica de uma micela reversa amplifica extraordinariamente a taxa de reação foto-redox devido à diminuição do volume livre dos reagentes. O radical sulfinato formado, sendo uma espécie neutra, tende a migrar para a fase apolar reduzindo a probabilidade de recombinação intramicelar, e dando condições para a reação de iniciação por adição ao monômero vinílico solubilizado na fase apolar.

Esta estratégia de confinamento molecular do sistema iniciador fotoquímico em micelas reversas foi aplicada na polimerização de estireno com obtenção de um polímero com peso molecular da ordem de $310.000 \mathrm{~g} / \mathrm{mol}$ e polidispersividade de 2,1 , valores estes determinados via cromatografia de permeação em gel $^{44}$.

\section{MÉTODOS EXPERIMENTAIS}

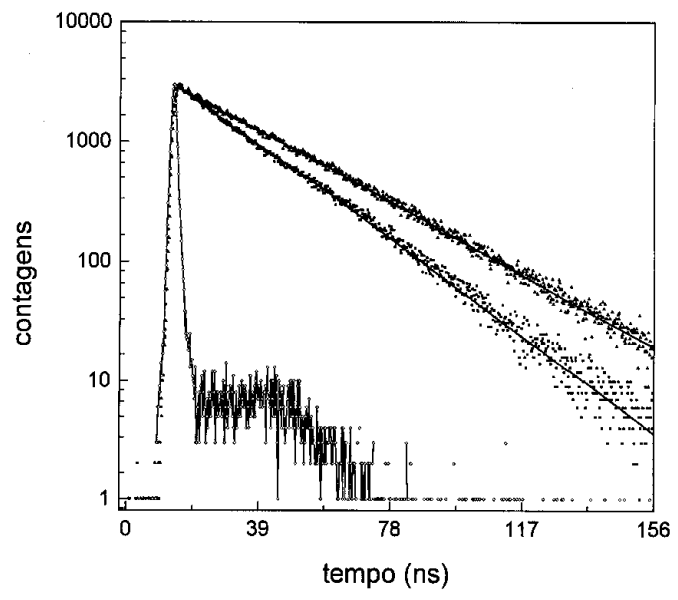

Figura 8. Decaimento de fluorescência do corante acridina obtido pela técnica de contagem de fótons, na ausência (4) e presença de divinilbenzeno (ロ, 0,5 mM) em metanol. Tempo de vida da acridina: 27,1 $\pm 0,2 \mathrm{~ns} ;$ constante de velocidade de supressão: $1,6 \pm 0,1 \times 10^{10} \mathrm{M}^{-1} \mathrm{~s}^{-1}$. - - - representa o pulso de excitação da amostra.

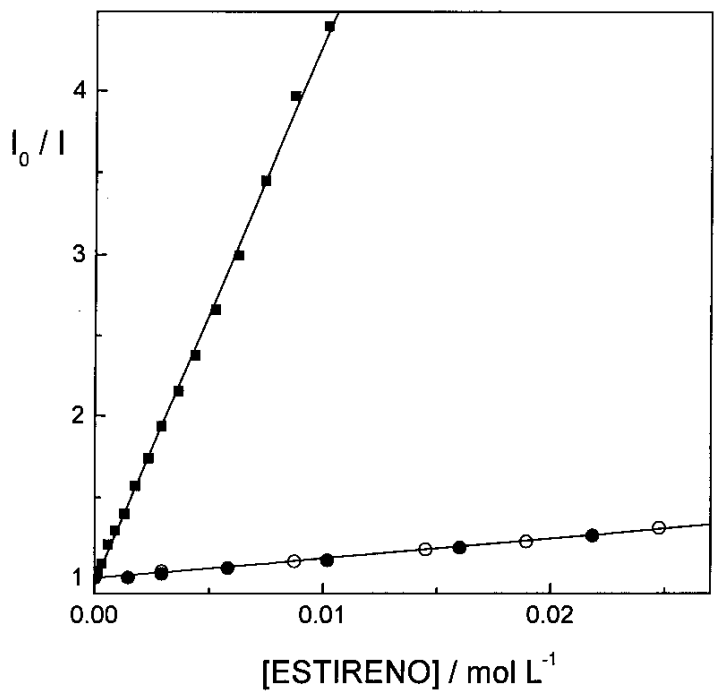

Figura 9. Gráfico de Stern-Volmer para a supressão de fluorescência de acridina por estireno em metanol (口) e em micelas reversas (SDS/hexanol em ciclohexano, $w=10)\left(\bullet, I_{0} / I\right) ;\left(O, \tau_{0} / \tau\right)$. 
Em geral as velocidades e taxas de fotopolimerização são estudadas usando os mesmos métodos que são usados para as polimerizações térmicas, mas mantendo alguns cuidados específicos necessários em sistemas submetidos a irradiação uv-visível.

A técnica tradicionalmente usada para medir polimerizações é a dilatometria, que está baseada no aumento de volume específico associado a reações de polimerização ${ }^{45}$. Quando se estudam fotopolimerizações ${ }^{30,38,46}$ deve se ter especial cuidado com a termostatização, já que pequenas variações de temperatura podem produzir mudanças bastante significativas na variação de altura no dilatômetro.

Outro método geralmente usado e que não oferece maiores dificuldades é a medida gravimétrica do polímero. Nestes casos, é necessário separar alíquotas do sistema reacional e precipitar o polímero, geralmente pelo agregado de algum não-solvente. A velocidade polimerização $v_{p}$ em mol/Ls é calculada a partir de ${ }^{47}$

$v_{p}=\frac{1000 W}{V \cdot t \cdot M_{w}}$

onde $W$ é a massa de polímero recuperada, $V$ o volume da alíquota, $t$ o tempo de reação e $\mathrm{M}_{\mathrm{w}}$ a massa molecular do monômero. Como a velocidade de polimerização varia com o avanço da reação é conveniente extrapolar a velocidade para tempo zero para poder ter valores significativos. Esta metodologia tem sido aplicada a sistemas homogêneos ${ }^{39}$ e micelares ${ }^{48}$. Uma aplicação interessante do método gravimétrico foi desenvolvida por Sudesh Kumar e Neckers ${ }^{49}$ para avaliar o rendimento quântico de fotopolimerização por laser.

Mais recentemente foram desenvolvidos outros métodos que utilizam tecnologias mais modernas. Para baixas taxas de conversão, o número de cadeias poliméricas presentes como partículas coloidais é proporcional à quantidade de monômero polimerizado. Como estas partículas espalham a luz, medidas da intensidade do espalhamento permitem avaliar o grau de avanço da reação, já que existe uma correlação direta entre a massa precipitada do polímero e o grau de espalhamento ${ }^{50}$.

O monitoramento da banda vibracional do grupo vinila na região de $800-820 \mathrm{~cm}^{-1}$ usando a espectroscopia de infravermelho por transformada de Fourier é outra forma de acompanhar a velocidade da polimerização ${ }^{51}$. Esta técnica é referida, às vezes, como RTIR (real-time infrared spectroscopy).

Em geral, as polimerizações são exotérmicas, o que permite um acompanhamento das reações medindo a evolução de calor em um microcalorímetro ${ }^{52}$. A variação da viscosidade e da micropolaridade a medida que avança a polimerização, são propriedades que podem ser aproveitadas para seguir a polimerização. Isto é a base da utilização de sondas fluorescentes que mudam suas propriedades espectroscópicas com a mudança do meio nas quais estão localizadas ${ }^{53}$.

\section{CONSIDERAÇÕES FINAIS E CONCLUSÕES}

A utilização de corantes em reações foto-redox com co-iniciadores compreende uma classe de sistemas fotoquímicos úteis para a geração de radicais livres ativos para polimerização de monômeros vinílicos, tanto em fase homogênea como em fase micro-heterogênea. $O$ prospecto deste artigo está limitado a uma discussão de sistemas investigados no Laboratório de Fotoquímica - IQSC ${ }^{@}$, porém vários outros sistemas envolvendo corantes tem sido descritos na literatura especializada ${ }^{7-12}$. Alguns dos aspectos centrais de pesquisa nesta área envolvem a otimização da polimerização para um aumento no percentual de conversão, visto que para alguns sistemas baseados em corantes os rendimentos de formação de polímero são relativamente baixos quando comparados aos processos térmicos. Por outro lado, é de interesse a utilização de formulações fotopolimerizáveis para usos biológicos ou biomédicos, onde deve evitar-se a presença de resíduos de coiniciadores relativamente tóxicos. Para isso deveriam estudar-se reações foto-redox com corantes em sistemas de baixa toxicidade, como p. ex., usando aminoácidos (fenilglicina e derivados) ou carboxilatos (ácido ascórbico).

Finalmente, um polímero obtido por meio de reações fotoquímicas envolvendo corantes não deve a priori apresentar a coloração do iniciador. Assim pesquisas na área de corantes suportados em sistemas como argilas, membranas poliméricas trocadoras de íons e sistemas de micro-encapsulamento constituem-se de novos campos de estudo, que permitem obter polímeros com maior estabilidade fotoquímica, além de recuperar os corantes iniciadores.

\section{AGRADECIMENTOS}

Os autores agradecem a FAPESP (94/3505), FINEP (65/ 92.0063.00) e CNPq pelo suporte financeiro.

\section{REFERÊNCIAS}

1. Bamford, C. H.; Radical Polymerization; Encyclopedia of Polymer Science and Engineering 2nd ed., J. Wiley \& Sons: New York 1989, vol. 13, p.708.

2. Reiser, A.; Photoreactive Polymers: The Science and Technology of Resists; J. Wiley \& Sons: New York 1989.

3. Fouassier, J. P.; Lougnot, D. J.; Radiation Curing of Polymeric Materials, ACS Symp. Ser. 417, vol. 5, 1990, p. 59.

4. Dietliker, R. K.; Chemistry and Technology of $U V$ and EB Formulation for Coatings, Inks, and Paints, SITA Technology Ltd.: London 1991, vol. 3, p. 59.

5. Fouassier, J. P; Rabek, J. F.; Lasers in Polymer Science and Technology: Applications, CRC Press: Boca Raton 1989.

6. Neckers, D. C.; Polymer Engineering and Science 1992, 32,1481 .

7. Gruber, H. F.; Prog. Polym. Sci. 1992, 17, 953.

8. Timpe, H. J.; Jockusch, S.; Körner, K.; Radiation Curing in Polymer Science and Technology ; Fouassier, J. P.; Rabek, J. F.; Eds.; Elsevier: London 1993, Vol. II, p. 575.

9. Eaton, D. F.; Advances in Photochemistry ; Volman, D.; Gollnick, K.; Hammond, G.; Eds.; Wiley: New York 1986, vol. 13, p. 427.

10. Eaton, D. F.; Topics in Current Chemistry; Mattay, J.; Ed.; Springer Verlag: Berlin 1990, vol. 156, p. 199.

11. Schuster, G. B.; Pure Appl. Chem. 1990, 62, 1565.

12. Monroe, B. H.; Weed, G. C.; Chem. Rev. 1993, 93, 435.

13. Lissi, E. A.; Encinas, M. V.; Photochemistry and Photophysics, Rabek, J. F.; Ed.; CRC Press: Boca Raton, FL 1991, vol. IV, p. 221.

14. El-Aasser, M. S.; Emulsion Polymerization; An Introduction to Polymer Colloids, Candau, F.; Ottewill, R. H.; Eds.; Kluwer Academic Publishers: Dordrecht 1990, p. 1.

15. Wang, Q.; Fu, S.; Yu, T.; Prog. Polym. Sci. 1994, 19, 703.

16. Candau, F.; Polymerization in Inverse Emulsions and Microemulsions; An Introduction to Polymer Colloids, Candau, F.; Ottewill, R. H.; Eds.; Kluwer Academic Publishers: Dordrecht 1990, p. 73.

17. Kuo, P.; Turro, N. J.; Tseng, C.; El-Aasser, M. S.; Vanderhoff, J. W.; Macromolecules 1987, 20, 1216.

18. Cochin, D.; Candau, F.; Zana, R.; Macromolecules 1993, 26, 5755 .

19. Cochin, D.; Zana, R.; Candau, F.; Macromolecules 1993, 26, 5765.

20. Smith, W. V.; Ewart, R. H.; J. Chem. Phys. 1948, 16, 592.

21. Gehlen, M. H.; De Schryver, F. C.; Chem. Rev. 1993 93, 199.

22. Gehlen, M. H.; J. Phys. Chem. 1995, 99, 4181.

23. O’Toole, J. T.; J. Appl. Polym. Sci. 1965, 9, 1291.

24. Napper, D. H.; Gilbert, R. G.; Emulsion Polymerization: The Mechanisms of Latex Particle Formation and Growth, 
An Introduction to Polymer Colloids, Candau, F.; Ottewill, R. H.; Eds.; Kluwer Academic Publishers: Dordrecht 1990, p. 159.

25. Hunkeler, D.; Hamielec, A. E.; Baade, W.; Polymer 1989, $30,127$.

26. Candau, F.; Leong, Y. S.; Fitch, R. M.; J. Polym. Sci. Polym. Chem. Ed. 1985, 23, 1903.

27. Carver, M.; Dreyer, U.; Kroesel, R.; Candau, F.; Fitch, R. M.; J. Polymer Sci. Polym. Ed. 1989, 27, 216.

28. Candau, F.; Zekhnini, Z.; Heatley, F.; Frauta, F.; Colloid Polym. Sci. 1986, 264, 676.

29. Morgan, J. D.; Lusvarki, K. M.; Kaler, E. W.; Macromolecules 1997, 30, 1897.

30. Previtali, C. M.; Bertolotti, S. G.; Neumann, M. G.; Pastre, I. A.; Rufs, A. M.; Encinas, M. V.; Macromolecules 1994, 27, 1994.

31. Neumann, M. G.; Hioka, N.; Appl. Polym. Sci. 1987, 43, 2828.

32. Neumann, M. G.; Gehlen, M. H.; J. Colloid Interface Sci. 1990, 135, 209.

33. Neumann, M. G.; Gessner, F.; Oliveira, V. A.; J. Chem. Soc. Faraday Trans. 1990, 86, 3551.

34. Neumann, M. G.; Scaiano, J. C.; J. Photochem. Photobiol. A: Chem. 1990, 54, 73.

35. Neumann, M. G.; Pastre, I. A.; Previtali, C. M.; J. Photochem. Photobiol. A: Chem. 1991, 61, 91.

36. Encinas, M. V.; Previtali, C. M. Bertolotti, S. G.; Neumann, M. G.; Photochem. Photobiol. 1995, 62, 64.

37. Encinas, M. V.; Previtali, C. M.; Gehlen, M. H.; Neumann, M. G.; J. Photochem. Photobiol. A: Chem. 1996, 94, 237.

38. Encinas, M. V.; Rufs, A. M.; Previtali, C. M.; Neumann, M. G.; Polymer 1995, 37, 1395.

39. Neumann, M. G.; Rodrigues, M. R.; Polymer 1998, 39, 1657.

40. Margerum, J. D.; Lackner, A. M.; Little, M. J.; Pretrusis,
C. T.; J. Phys. Chem. 1971, 75, 3066.

41. Margerum, J. D.; Brault, R. G.; Lackner, A. M.; Miller, L. J.; J. Phys. Chem. 1973, 77, 2720.

42. Miller, L. J.; Margerum, J. D.; Rust, J. B.; Brault, R. G.; Lackner, A. M.; Macromolecules 1974, 7, 179.

43. Buchviser, S. F.; Gehlen, M. H.; J. Chem. Soc. Faraday Trans. 1997, 93, 1133.

44. Buchviser, S. F.; Gehlen, M. H.; Proc. Eighth Inter. Am. Photochem. Soc. Conf.; Foz do Iguaçu 1996, p. 6.

45. Billmeyer Jr., F. W.; Textobook of Polymer Science, $3^{\mathrm{a}}$. Ed., Wiley, New York, 1984; Collins, E. A.; Bares, J.; Billmeyer Jr., F.W.; Experiments in Polymer Science, Wiley-Interscience, New York, 1973.

46. Block, H.; Ledwith, A.; Taylor, A. R.; Polymer 1971, 12,271

47. Lenka, S; Mohanty, I. B.; J. Polym. Sci., Polym. Chem. Ed. 1986, 2224, 2729.

48. Merlin, A.; Fouassier, J. P.; Polymer 1980, 21, 1363.

49. Sudesh Kumar, G.; Neckers, D. C.; Macromolecules 1991, $24,4322$.

50. Rust, J. B.; Miller, L. J.; Margerum, J. D.; Polym. Eng. Sci. 1969, 9, 40.

51. Decker, C.; Moussa, K.; Makromol. Chem. 1988, 189, 2381; Decker, C.; Moussa, K.; Macromolecules 1989, 22, 4455.

52. Paczkowski, J.; Pietrzak, M.; Kucybala, Z.; Macromolecules 1996, 29, 5057.

53. Paczkowski, J.; Neckers, D. C.; J. Polym. Sci., Part A: Polym. Chem. 1993, 31, 841; Winnik, M. A.; Photophysical and Photochemical Tools in Polymer Science, NATO, AS Series Ed., Reidel: Dordrecht 1986. 